\title{
IUCrJ
}

Volume 6 (2019)

Supporting information for article:

Supramolecular synthon hierarchy in sulfonamide cocrystals with syn-amides and $\mathrm{N}$-oxides

Geetha Bolla and Ashwini Nangia 


\section{X-ray powder diffraction}

Bulk samples were analyzed by X-ray powder diffraction on a Bruker AXS D8 diffractometer (BrukerAXS, Karlsruhe, Germany). Experimental conditions: $\mathrm{Cu}$ K radiation $(\lambda=1.54056 \AA), 40 \mathrm{kV}, 30 \mathrm{~mA}$, scanning interval 5-50 $2 \theta$.

\section{Thermal analysis Differential Scanning Calorimetry (DSC)}

DSC was performed on a Mettler Toledo DSC 822e module. Samples were placed in crimped but vented aluminium sample pans, with a typical sample weighed of 3-5 mg. The temperature range was set 30 $200{ }^{\circ} \mathrm{C} \min ^{-1}$ at a heating rate of $10{ }^{\circ} \mathrm{C} \mathrm{min}$. While sample running it was purged with a stream of dry

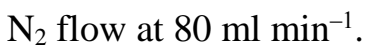

Table S1 Hydrogen bond geometry of the crystal structures in this present study.

\begin{tabular}{|c|c|c|c|c|c|}
\hline Crystal & Interaction & $\mathrm{H} \cdots \mathrm{A} / \AA$ & $\mathrm{D} \cdots \mathrm{A} / \mathrm{A}$ & $\angle \mathrm{D}-\mathrm{H} \cdots \mathrm{A} /{ }^{\circ}$ & Symmetry code \\
\hline \multirow{8}{*}{$\begin{array}{c}\text { CEL-2HP } \\
(1: 1)\end{array}$} & 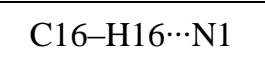 & 3.01 & $3.376(4)$ & 105 & $1-X,-Y,-Z$ \\
\hline & $\mathrm{C} 10-\mathrm{H} 10 \cdots \mathrm{F} 3$ & 2.59 & $3.321(4)$ & 136 & $1-X, 1-Y,-Z$ \\
\hline & $\mathrm{N} 3-\mathrm{H} 3 \mathrm{~A} \cdots \mathrm{O} 3$ & $2.06(3)$ & $2.925(4)$ & 171 & $1 / 2+X, 1 / 2-Y,-1 / 2+Z$ \\
\hline & $\mathrm{N} 3-\mathrm{H} 3 \mathrm{~B} \cdots \mathrm{O} 2$ & $2.22(3)$ & $3.072(4)$ & 166 & $3 / 2-X, 1 / 2+Y,-1 / 2-Z$ \\
\hline & $\mathrm{C} 11-\mathrm{H} 11 \mathrm{~A} \cdots \mathrm{N} 1$ & 2.79 & $3.464(4)$ & 127 & $1 / 2+X, 1 / 2-Y, 1 / 2+Z$ \\
\hline & $\mathrm{N} 4-\mathrm{H} 4 \cdots \mathrm{F} 1$ & 2.80 & $3.092(3)$ & 101 & $1 / 2+\mathrm{X}, 1 / 2-\mathrm{Y}, 1 / 2+\mathrm{Z}$ \\
\hline & $\mathrm{N} 4-\mathrm{H} 4 \cdots \mathrm{O} 3$ & 1.94 & $2.800(4)$ & 178 & $1-X,-Y, 1-Z$ \\
\hline & $\mathrm{C} 19-\mathrm{H} 19 \cdots \mathrm{O} 1$ & 2.59 & $3.492(5)$ & 162 & $-1 / 2+X, 1 / 2-Y, 1 / 2+Z$ \\
\hline \multirow{3}{*}{ CEL-MeHP } & $\mathrm{N} 4-\mathrm{H} 4 \mathrm{~A} \cdots \mathrm{O} 2$ & 2.18 & 2.994(3) & 157 & $1-X, 2-Y, 1-Z$ \\
\hline & $\mathrm{N} 1-\mathrm{H} 1 \cdots \mathrm{O} 1$ & 1.97 & $2.823(3)$ & 178 & $-\mathrm{X}, 2-\mathrm{Y},-\mathrm{Z}$ \\
\hline & $\mathrm{N} 4-\mathrm{H} 4 \mathrm{~B} \cdots \mathrm{O} 1$ & 2.09 & $2.882(3)$ & 173 & $-\mathrm{X}, 2-\mathrm{Y},-\mathrm{Z}$ \\
\hline \multirow{4}{*}{$\begin{array}{c}\text { CEL- } \\
\text { OMeHP } \\
(1: 1)\end{array}$} & $\mathrm{C} 16-\mathrm{H} 16 \cdots \mathrm{O} 2$ & 2.53 & $2.897(3)$ & 104 & $1-X, 2-Y, 1-Z$ \\
\hline & $\mathrm{N} 3-\mathrm{H} 3 \mathrm{~A} \cdots \mathrm{O} 1$ & 2.23 & $2.987(3)$ & 167 & $-X,-Y,-Z$ \\
\hline & N3-H3B $\cdots \mathrm{O} 3$ & 2.07 & 2.893(3) & 161 & $1+\mathrm{X},-1+\mathrm{Y},+\mathrm{Z}$ \\
\hline & $\mathrm{N} 4-\mathrm{H} 4 \cdots \mathrm{O} 3$ & 1.91 & $2.800(3)$ & 175 & $-X, 1-Y,-Z$ \\
\hline \multirow{3}{*}{$\begin{array}{c}\text { CEL- } \\
\text { MeTFHP } \\
(1: 1)\end{array}$} & $\mathrm{N} 1-\mathrm{H} 1 \cdots \mathrm{O} 1$ & 1.97 & $2.823(3)$ & 178 & $1-X, 1-Y,-Z$ \\
\hline & $\mathrm{N} 4-\mathrm{H} 4 \mathrm{~B} \cdots \mathrm{O} 1$ & 2.09 & $2.882(3)$ & 173 & $1 / 2+X, 1 / 2-Y,-1 / 2+Z$ \\
\hline & $\mathrm{N} 4-\mathrm{H} 4 \mathrm{~A} \cdots \mathrm{O} 2$ & 2.18 & 2.994(3) & 157 & $1-X,-Y,-Z$ \\
\hline \multirow{5}{*}{$\begin{array}{c}\text { HCT-VLM } \\
\quad(1: 2)\end{array}$} & $\mathrm{C} 16-\mathrm{H} 16 \mathrm{~B} \cdots \mathrm{O} 1$ & 2.75 & $3.2364(18)$ & 114 & $+\mathrm{X},+\mathrm{Y}, 1+\mathrm{Z}$ \\
\hline & $\mathrm{C} 15-\mathrm{H} 15 \mathrm{~B} \cdots \mathrm{O} 4$ & 2.65 & $3.3388(19)$ & 126 & $2-X, 1-Y, 1-Z$ \\
\hline & $\mathrm{N} 5-\mathrm{H} 5 \cdots \mathrm{O} 4$ & 2.36 & $3.1681(16)$ & 159 & $+\mathrm{X}, 1+\mathrm{Y}, 1+\mathrm{Z}$ \\
\hline & $\mathrm{N} 4-\mathrm{H} 4 \mathrm{~A} \cdots \mathrm{O} 5$ & 2.08 & $2.9055(17)$ & 177 & $1-X, 1-Y,-Z$ \\
\hline & $\mathrm{N} 2-\mathrm{H} 2 \cdots \mathrm{O} 3$ & 2.21 & $2.9148(16)$ & 142 & $2-X, 1-Y, 1-Z$ \\
\hline
\end{tabular}




\begin{tabular}{|c|c|c|c|c|c|}
\hline & $\mathrm{N} 1-\mathrm{H} 1 \cdots \mathrm{O} 6$ & 2.03 & $2.8357(17)$ & 172 & $2-X, 1-Y, 1-Z$ \\
\hline & 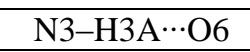 & 2.17 & $2.9508(17)$ & 154.4 & $1-X, 1-Y, 1-Z$ \\
\hline \multirow{7}{*}{$\begin{array}{l}\mathrm{HCT}-\mathrm{CPR} \\
\quad(1: 2)\end{array}$} & $\mathrm{C} 4-\mathrm{H} 4 \cdots \mathrm{O} 5$ & 2.55 & $3.303(2)$ & 137 & $1 / 2+X, 1 / 2-Y, 1-Z$ \\
\hline & C9-H9B $\cdots \mathrm{O} 1$ & 2.84 & $3.554(2)$ & 131 & $1-X, 1 / 2+Y, 3 / 2-Z$ \\
\hline & $\mathrm{N} 4-\mathrm{H} 4 \mathrm{~A} \cdots \mathrm{O} 3$ & 2.21 & $2.9831(19)$ & 169 & $-1+X,+Y,+Z$ \\
\hline & $\mathrm{N} 1-\mathrm{H} 1 \cdots \mathrm{O} 5$ & 2.20 & $2.943(2)$ & 154 & $1 / 2+X, 1 / 2-Y, 1-Z$ \\
\hline & $\mathrm{N} 5-\mathrm{H} 5 \cdots \mathrm{O} 1$ & 2.21 & $3.024(2)$ & 176 & $1-X,-Y, 1-Z$ \\
\hline & $\mathrm{N} 2-\mathrm{H} 2 \cdots \mathrm{O} 6$ & 2.00 & $2.811(2)$ & 154 & $1+X,+Y,+Z$ \\
\hline & N3-H3A $\cdots$ O6 & 2.08 & $2.917(2)$ & 171 & $1-X,-Y, 1-Z$ \\
\hline \multirow{12}{*}{$\begin{array}{c}\text { HCT-2HP } \\
\text { Form I } \\
(1: 1)\end{array}$} & $\mathrm{C} 4-\mathrm{H} 4 \cdots \mathrm{O} 5$ & 2.55 & $3.303(2)$ & 137 & $1+X, 3 / 2-Y, 1 / 2+Z$ \\
\hline & $\mathrm{C} 7-\mathrm{H} 7 \mathrm{~A} \cdots \mathrm{O} 3$ & 2.68 & $3.025(2)$ & 101 & $1-X, 1 / 2+Y, 1 / 2-Z$ \\
\hline & $\mathrm{C} 13-\mathrm{H} 13 \mathrm{~A} \cdots \mathrm{O} 2$ & 2.52 & $3.483(2)$ & 175 & $2-X,-1 / 2+Y, 1 / 2-Z$ \\
\hline & $\mathrm{C} 18-\mathrm{H} 18 \mathrm{~A} \cdots \mathrm{O} 5$ & 3.00 & $3.473(3)$ & 111 & $2-X, 2-Y,-Z$ \\
\hline & $\mathrm{N} 4-\mathrm{H} 4 \mathrm{~A} \cdots \mathrm{O} 3$ & 2.21 & $2.9831(19)$ & 169 & $2-X, 1-Y, 1-Z$ \\
\hline & $\mathrm{N} 1-\mathrm{H} 1 \cdots \mathrm{O} 5$ & 2.20 & $2.943(2)$ & 154 & $1+X, 3 / 2-Y, 1 / 2+Z$ \\
\hline & $\mathrm{N} 1-\mathrm{H} 1 \cdots \mathrm{O} 4$ & 2.88 & $3.2023(19)$ & 106 & $1-\mathrm{X}, 1 / 2+\mathrm{Y}, 1 / 2-\mathrm{Z}$ \\
\hline & $\mathrm{N} 5-\mathrm{H} 5 \cdots \mathrm{O} 1$ & 2.21 & $3.024(2)$ & 176 & $+\mathrm{X},-1+\mathrm{Y}, 1+\mathrm{Z}$ \\
\hline & $\mathrm{N} 2-\mathrm{H} 2 \cdots \mathrm{O} 6$ & 2.00 & $2.811(2)$ & 154 & $-1+\mathrm{X}, 3 / 2-\mathrm{Y}, 1 / 2+\mathrm{Z}$ \\
\hline & N3-H3A $\cdots$ O6 & 2.08 & $2.917(2)$ & 171 & $+\mathrm{X},-1+\mathrm{Y}, 1+\mathrm{Z}$ \\
\hline & 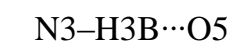 & 2.05 & $2.847(2)$ & 167 & $+X, 3 / 2-Y, 1 / 2+Z$ \\
\hline & $\mathrm{C} 4-\mathrm{H} 4 \cdots \mathrm{O} 1$ & 2.455 & $3.358(10)$ & 163 & $1+\mathrm{X}, 3 / 2-\mathrm{Y}, 1 / 2+\mathrm{Z}$ \\
\hline \multirow{6}{*}{$\begin{array}{c}\text { HCT-2HP } \\
\text { Form II } \\
(1: 1)\end{array}$} & $\mathrm{N} 2-\mathrm{H} 2 \cdots \mathrm{O} 2$ & 3.10 & $3.463(9)$ & 111 & $+\mathrm{X},+\mathrm{Y},-1+\mathrm{Z}$ \\
\hline & N3-H3A $\cdots$ O4 & 2.23 & $2.971(8)$ & 166 & $2-X, 1-Y,-1 / 2+Z$ \\
\hline & N3-H3B $\cdots \mathrm{O} 3$ & 1.88 & $2.849(8)$ & 168 & $2-X, 2-Y,-1 / 2+Z$ \\
\hline & $\mathrm{C} 9-\mathrm{H} 9 \cdots \mathrm{O} 1$ & 2.661 & $3.326(11)$ & 129 & $3 / 2 \mathrm{X}, 1 / 2+\mathrm{Y}, 1 / 2+\mathrm{Z}$ \\
\hline & $\mathrm{C} 11-\mathrm{H} 11 \cdots \mathrm{O} 3$ & 2.665 & $3.560(13)$ & 161 & $\begin{array}{c}3 / 2-\mathrm{X}, 1 / 2+\mathrm{Y},-1 / 2+ \\
\mathrm{Z}\end{array}$ \\
\hline & $\mathrm{N} 4-\mathrm{H} 4 \mathrm{~A} \cdots \mathrm{N} 2$ & 2.6 & $3.174(14)$ & 127 & $+\mathrm{X},-1+\mathrm{Y},+\mathrm{Z}$ \\
\hline \multirow{7}{*}{$\begin{array}{l}\text { FUROS- } \\
\text { 2PYM } \\
(2: 2: 1)\end{array}$} & N1-H1A $\cdots$ O6 & 2.43 & $3.437(6)$ & 150 & $+X,-Y,-1 / 2+Z$ \\
\hline & 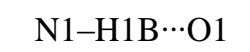 & 2.82 & $3.000(5)$ & 137 & $1 / 2-X, 0.5-Y, Z$ \\
\hline & $\mathrm{N} 3-\mathrm{H} 3 \mathrm{~A} \cdots \mathrm{O} 3$ & 2.12 & $2.929(4)$ & 155 & $-\mathrm{X},+\mathrm{Y}, 3 / 2-\mathrm{Z}$ \\
\hline & $\mathrm{C} 12-\mathrm{H} 12 \cdots \mathrm{O} 1$ & 2.59 & $3.483(6)$ & 160 & $1 / 2-\mathrm{X}, 1 / 2+\mathrm{Y}, 1 / 2-\mathrm{Z}$ \\
\hline & $\mathrm{C} 17-\mathrm{H} 17 \mathrm{~B} \cdots \mathrm{N} 2$ & 2.44 & $3.356(6)$ & 159 & $-X, 1-Y, 1-Z$ \\
\hline & N1-H1A $\cdots$ O6 & 2.82 & $3.437(6)$ & 150 & $+X,-Y,-1 / 2+Z$ \\
\hline & $\mathrm{N} 1-\mathrm{H} 1 \mathrm{~B} \cdots \mathrm{O} 1$ & 2.43 & $3.000(5)$ & 137 & $1 / 2-X, 1 / 2-Y,-Z$ \\
\hline \multirow{3}{*}{$\begin{array}{c}\text { FUROS- } \\
\text { CPR } \\
(1: 1)\end{array}$} & $\mathrm{N} 3-\mathrm{H} 3 \cdots \mathrm{O} 1$ & 2.28 & $3.016(3)$ & 143 & $1-X, 2-Y, 2-Z$ \\
\hline & $\mathrm{C} 7-\mathrm{H} 7 \cdots \mathrm{O} 2$ & 2.42 & $2.839(3)$ & 107 & $1-X, 1-Y, 1-Z$ \\
\hline & 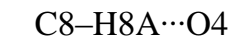 & 2.63 & $3.359(3)$ & 132 & $1+X,+Y,+Z$ \\
\hline
\end{tabular}




\begin{tabular}{|c|c|c|c|c|c|}
\hline & $\mathrm{C} 10-\mathrm{H} 10 \cdots \mathrm{O} 2$ & 2.52 & $3.274(5)$ & 138 & $1+X,+Y,+Z$ \\
\hline & $\mathrm{C} 12-\mathrm{H} 12 \cdots \mathrm{O} 1$ & 2.64 & $3.398(5)$ & 139 & $2-X, 1-Y, 2-Z$ \\
\hline & $\mathrm{N} 1-\mathrm{H} 1 \mathrm{~A} \cdots \mathrm{O} 3$ & 2.17 & $2.966(4)$ & 152 & $1-X, 2-Y, 2-Z$ \\
\hline & $\mathrm{N} 1-\mathrm{H} 1 \mathrm{~A} \cdots \mathrm{O} 6$ & 2.54 & $3.067(4)$ & 120 & $1-X, 2-Y, 1-Z$ \\
\hline & $\mathrm{N} 2-\mathrm{H} 2 \cdots \mathrm{O} 3$ & 2.03 & $2.660(3)$ & 143 & $1-X, 2-Y, 2-Z$ \\
\hline & $\mathrm{N} 1-\mathrm{H} 1 \mathrm{~B} \cdots \mathrm{O} 1$ & 2.44 & $3.163(4)$ & 157 & $1-X, 2-Y, 1-Z$ \\
\hline & $\mathrm{O} 4-\mathrm{H} 4 \cdots \mathrm{O} 6$ & 1.55 & $2.575(3)$ & 174 & $+X,+Y, 1+Z$ \\
\hline \multirow{11}{*}{$\begin{array}{c}\text { FUROS- } \\
\text { VLMH } \\
(1: 1: 1)\end{array}$} & $\mathrm{N} 2-\mathrm{H} 2 \cdots \mathrm{O} 3$ & 2.04 & $2.673(4)$ & 129 & $3 / 2-\mathrm{X}, 1 / 2+\mathrm{Y}, 1 / 2-\mathrm{Z}$ \\
\hline & $\mathrm{N} 2-\mathrm{H} 2 \cdots \mathrm{O} 7$ & 2.79 & $3.379(6)$ & 127 & $+\mathrm{X}, 1+\mathrm{Y},+\mathrm{Z}$ \\
\hline & 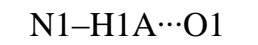 & 2.22 & $3.057(5)$ & 161 & $2-X, 1-Y,-Z$ \\
\hline & $\mathrm{N} 1-\mathrm{H} 1 \mathrm{~B} \cdots \mathrm{O} 7$ & 2.03 & $2.940(6)$ & 171 & $3 / 2-\mathrm{X}, 1 / 2+\mathrm{Y}, 1 / 2-\mathrm{Z}$ \\
\hline & $\mathrm{C} 8-\mathrm{H} 8 \mathrm{~A} \cdots \mathrm{O} 7$ & 2.94 & $2.675(6)$ & 102 & $+\mathrm{X}, 1+\mathrm{Y},+\mathrm{Z}$ \\
\hline & $\mathrm{C} 8-\mathrm{H} 8 \mathrm{~A} \cdots \mathrm{O} 4$ & 2.54 & $2.812(5)$ & 108 & $+X, 1+Y,+Z$ \\
\hline & $\mathrm{C} 17-\mathrm{H} 17 \mathrm{~B} \cdots \mathrm{N} 1$ & 2.81 & $2.977(5)$ & 164 & $3 / 2-\mathrm{X}, 1 / 2+\mathrm{Y}, 1 / 2-\mathrm{Z}$ \\
\hline & $\mathrm{C} 10-\mathrm{H} 10 \cdots \mathrm{O} 2$ & 2.75 & $3.288(5)$ & 133 & $+\mathrm{X}, 1+\mathrm{Y},+\mathrm{Z}$ \\
\hline & $\mathrm{C} 12-\mathrm{H} 12 \cdots \mathrm{O} 1$ & 2.39 & $3.494(6)$ & 117 & $5 / 2-\mathrm{X}, 1 / 2+\mathrm{Y}, 1 / 2-\mathrm{Z}$ \\
\hline & O7- H7B $\cdots$ O5 & 2.67 & $3.459(6)$ & 103 & $+\mathrm{X},-1+\mathrm{Y},+\mathrm{Z}$ \\
\hline & $\mathrm{O} 7-\mathrm{H} 7 \mathrm{~B} \cdots \mathrm{O} 7$ & 2.84 & $3.494(7)$ & 128 & $2-X,-Y, 1-Z$ \\
\hline
\end{tabular}

Table S2 Molecular structures of the all the components in this study.

Sulfonamide's




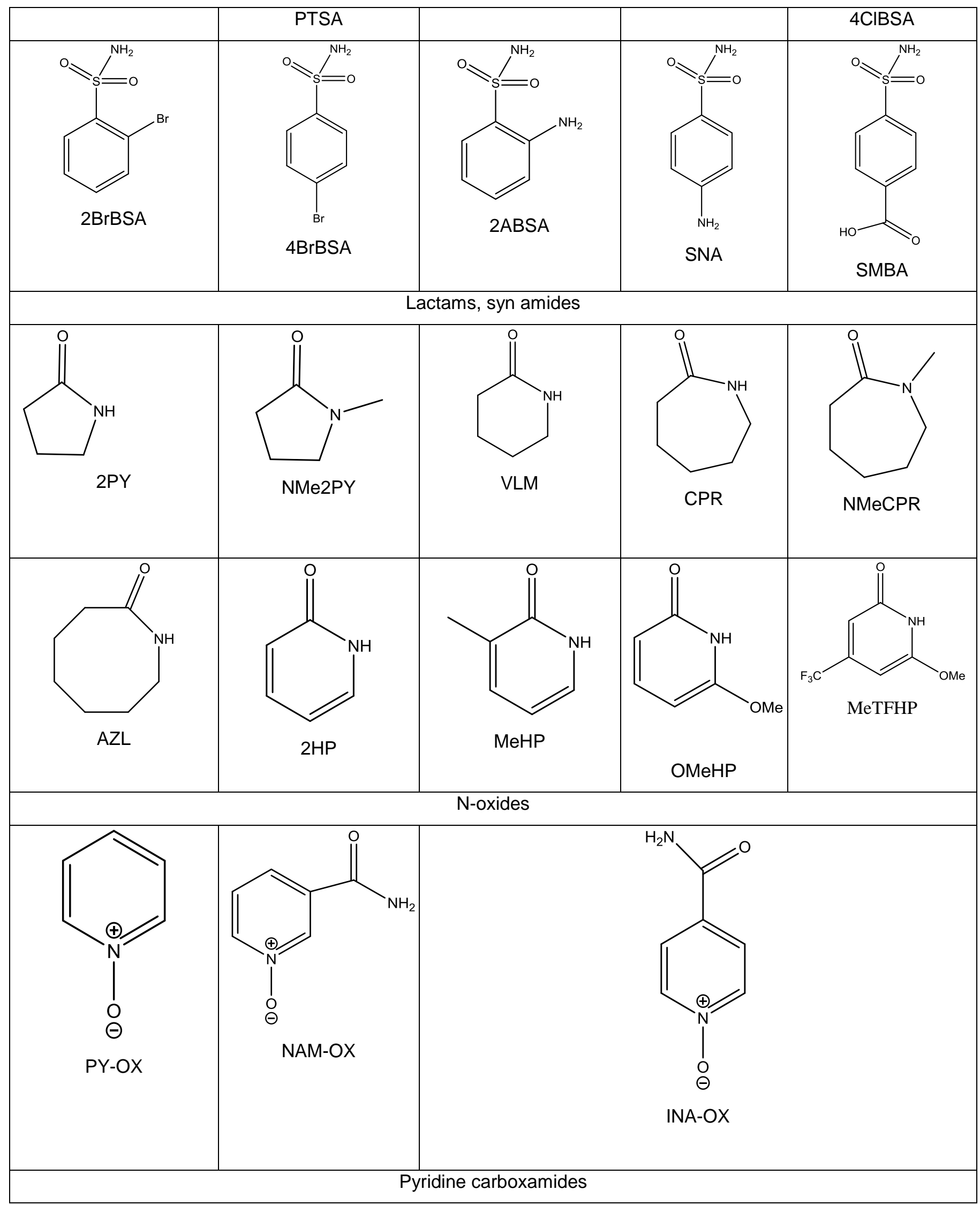




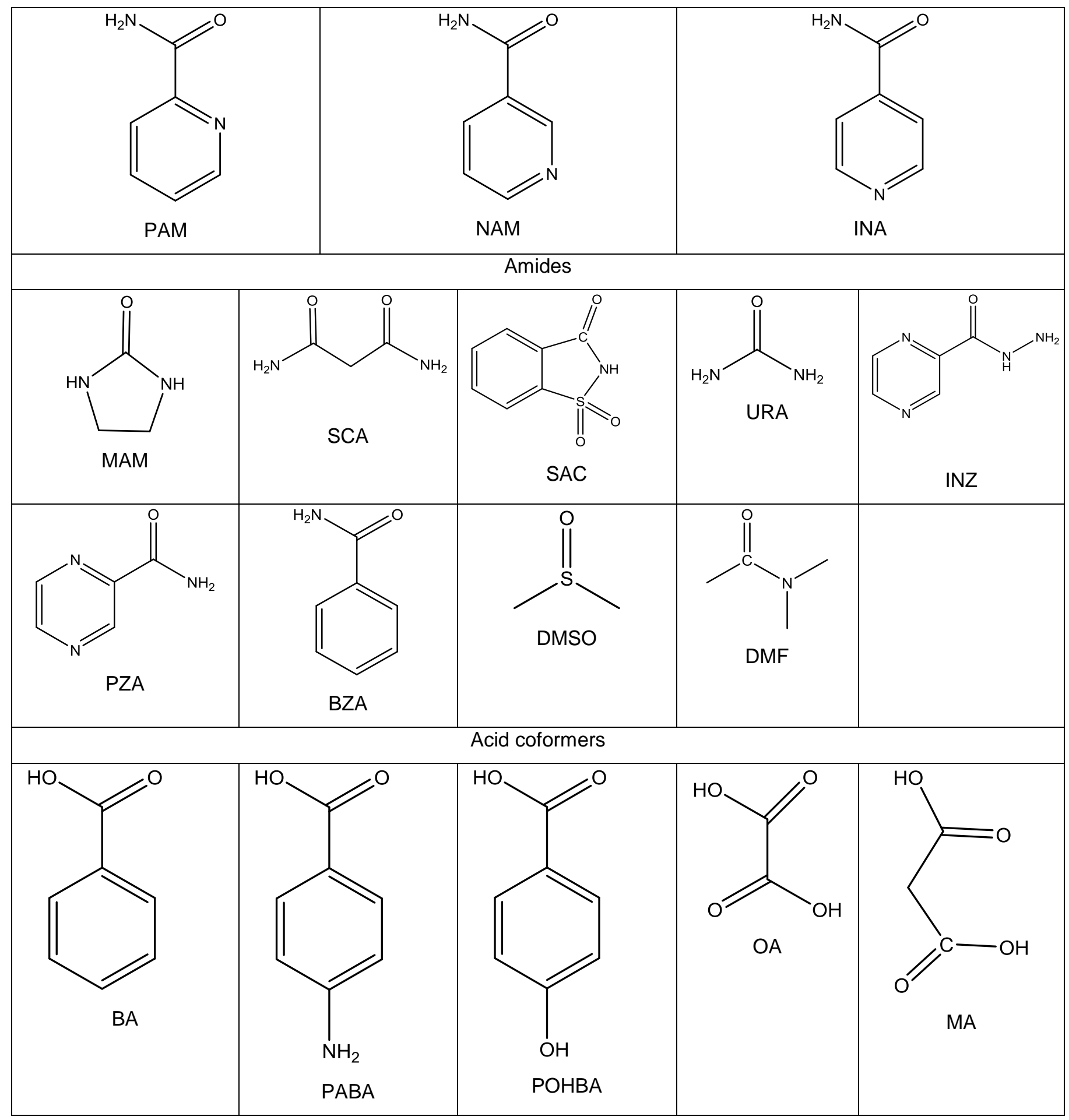

Table S3 Molecular electrostatic potentials energy calculations of sulphonamides and coformers using Spartan Student v7 software, Wavefunction, Inc., https://www.wavefun.com/ dated 27-01-2018. The calculated MEPE values are listed.

\begin{tabular}{|c|c|c|c|c|}
\hline \multirow{3}{*}{ Molecule } & \multicolumn{4}{|c|}{ DFT, 6-311+G** } \\
\cline { 2 - 5 } & $\begin{array}{c}\mathrm{E}, \mathrm{kJ} / \mathrm{mol} \\
\text { (gas) }\end{array}$ & $\begin{array}{c}\mathrm{E}, \mathrm{kJ} / \mathrm{mol} \\
\text { (water) }\end{array}$ & $\mathrm{E}, \mathrm{kJ} / \mathrm{mol}$ (polar) & $\mathrm{E}, \mathrm{kJ} / \mathrm{mol}$ (non-polar) \\
& & (DMF) & (THF) \\
\hline
\end{tabular}




\begin{tabular}{|c|c|c|c|c|}
\hline \multicolumn{5}{|c|}{ Sulfonamide } \\
\hline BSA & $-174.36,-216.11$ & $-220.59,-264.68$ & $-219.87,-264.02$ & $-212.57,-256.63$ \\
\hline PTSA & $-178.87,-210.68$ & $-227.479,-256.699$ & $-226.729-, 256.041$ & $-219.305,-249.151$ \\
\hline OTSA & $-177.055,-217.295$ & $-233.11,-279.359$ & $-232.125,-278.284$ & $-222.045,-266.897$ \\
\hline 2ClBSA & $-172.322,-223.214$ & $-222.86,-271.451$ & $-221.671,-270.723$ & $-213.94,-263.492$ \\
\hline $4 \mathrm{ClBSA}$ & $-162.49,-229.227$ & $-204.554,-280.837$ & $-203.953,-280.107$ & $-197.774,-272.292$ \\
\hline $2 \mathrm{BrBSA}$ & $-172.012,-223.592$ & $-228.469,-282.67$ & $-227.09,-281.268$ & $-214.711,-265.272$ \\
\hline 4BrBSA & $-162.772,-229.021$ & $-204.264,-281.333$ & $-203.626,-280.591$ & $-197.221,-272.946$ \\
\hline $2 \mathrm{ABSA}$ & $-182.857,-224.135$ & $-244.339,-278.208$ & $-243.335,-277.289$ & $-233.439,-269.164$ \\
\hline SNA & $-199.974,-236.632$ & $-269.138,-295.556$ & $-267.81,-294.541$ & $-257.213,-285.933$ \\
\hline SMBA & $-170.789,-293.551$ & $-224.97,-349.239$ & $-224.155,-348.356$ & $-216.016,-340.781$ \\
\hline \multicolumn{5}{|c|}{ Coformer } \\
\hline \multicolumn{5}{|c|}{ Lactam/syn-amide } \\
\hline $2 \mathrm{PY}$ & $-218.848,-195.349$ & $-283.399,-235.02$ & $-282.361,-234.444$ & $-273.377,-228.659$ \\
\hline NMePY & $-222.485,-94.4617$ & $-287.427,-130.077$ & $-286.328,-129.441$ & $-276.992,-123.688$ \\
\hline VLM & $-225.43,-176.87$ & $-291.41,-211.81$ & $-290.31,-211.30$ & $-280.984,-205.703$ \\
\hline CPR & $-224.175,-178.749$ & $-288.683,-211.988$ & $-287.62,-211.547$ & $-278.301,-206.610$ \\
\hline$\overline{A Z L}$ & $-225.684,-169.133$ & $-291.766,-200.701$ & $-290.760,-200.260$ & $-281.307,-195.271$ \\
\hline NMeCPR & $-225.291,-91.2207$ & $-293.083,-126.510$ & $-291.988,-125.872$ & $-282.269,-119.762$ \\
\hline $2 \mathrm{HP}$ & $-221.826,-234.175$ & $-291.149,-285.17$ & $-291.200,-285.163$ & $-280.897,-277.665$ \\
\hline MeHP & $-217.892,-227.594$ & $-287.264,-275.934$ & $-286.07,-275.198$ & $-275.723,-268.169$ \\
\hline OMeHP & $-230.326,-200.407$ & $-305.811,-223.092$ & $-304.467,-222.800$ & $-292.978,-220.097$ \\
\hline \multicolumn{5}{|c|}{ N-oxides } \\
\hline Py-oxide & $-202.200,-133.160$ & $-275.530,-173.830$ & $-274.160,-172.940$ & $-263.210,-166.800$ \\
\hline INA-oxide & $-180.989,-264.513$ & $-248.482,-368.555$ & $-247.186,-366.616$ & $-236.841,-351.385$ \\
\hline NAM-oxide & $-186.673,-257.840$ & $-248.276,-333.978$ & $-247.135,-332.502$ & $-238.242,-321.215$ \\
\hline \multicolumn{5}{|c|}{ Pyridine carboxamides } \\
\hline
\end{tabular}




\begin{tabular}{|c|c|c|c|c|}
\hline PAM & $-239.812,-230.451$ & $-354.678,-320.601$ & $-352.695,-318.947$ & $-334.594,-304.436$ \\
\hline NAM & $-190.997,-248.609$ & $-241.210,-317.000$ & $-240.580,-315.590$ & $-243.558,-325.721$ \\
\hline INA & $-181.132,-252.989$ & $-234.843,-339.711$ & $-233.882,-338.03$ & $-226.108,-325.090$ \\
\hline CYT & $-257.097,-252.969$ & $-353.516,-336.000$ & $-351.627,-334.486$ & $-337.173,-321.716$ \\
\hline \multicolumn{5}{|c|}{ Amides } \\
\hline Succinamide & $-160.923,-241.130$ & $-195.284,-273.644$ & $-194.743,-273.230$ & $-190.260,-269.184$ \\
\hline Malanomide & $-203.242,-264.010$ & $-254.074,-331.425$ & $-253.321,-330.305$ & $-246.485,-320.653$ \\
\hline Saccharin & $-147.536,-269.902$ & $-181.130,-313.545$ & $-180.603,-312.983$ & $-175.556,-307.571$ \\
\hline Urea & $-211.196,-224.404$ & $-280.719,-305.093$ & $-279.546,-303.676$ & $-269.024,-290.924$ \\
\hline Isoniazid & $-204.515,-231.339$ & $-281.88,-304.176$ & $-280.372,-302.718$ & $-269.104,-291.071$ \\
\hline Pyrazinamide & $-216.332,-248.060$ & $-305.861,-337.249$ & $-304.150,-335.490$ & $-290.678,-320.299$ \\
\hline Benzamide & $-201.241,-228.280$ & $-264.826,-303.390$ & $-263.622,-301.938$ & $-254.394,-291.108$ \\
\hline DMSO & $-233.437,-143.003$ & $-304.495,-205.536$ & $-303.314,-204.403$ & $-293.336,-194.880$ \\
\hline $\begin{array}{l}\text { NN-Dimethyl } \\
\text { acetamide }\end{array}$ & $-220.179,-113.021$ & $-285.157,-151.663$ & $-284.081,-150.943$ & $-274.347,-145.121$ \\
\hline $\begin{array}{c}\text { Tri phenyl } \\
\text { phosphine } \\
\text { Oxide }\end{array}$ & $-259.870,-121.315$ & $-334.280,-188.836$ & $-333.061,-187.748$ & $-322.491,-177.221$ \\
\hline \multicolumn{5}{|c|}{ Acid coformers } \\
\hline $\mathrm{BA}$ & $-163.486,-259.511$ & $-200.798,-303.011$ & $-200.197,-302.237$ & $-194.896,-296.150$ \\
\hline PABA & $-184.949,-234.693$ & $-235.158,-279.374$ & $-234.297,-278.353$ & $-226.37,-269.255$ \\
\hline POHBA & $-168.829,-296.294$ & $-207.394,-365.022$ & $-206.762,-363.880$ & $-201.205,-354.800$ \\
\hline Oxalic acid & $-149.224,-307.900$ & $-176.319,-373.677$ & $-175.837,-372.804$ & $-172.537,-364.657$ \\
\hline Melonic Acid & $-154.38,-284.829$ & $-215.735,-335.212$ & $-214.102-, 334.34$ & $-202.640,-327.206$ \\
\hline
\end{tabular}




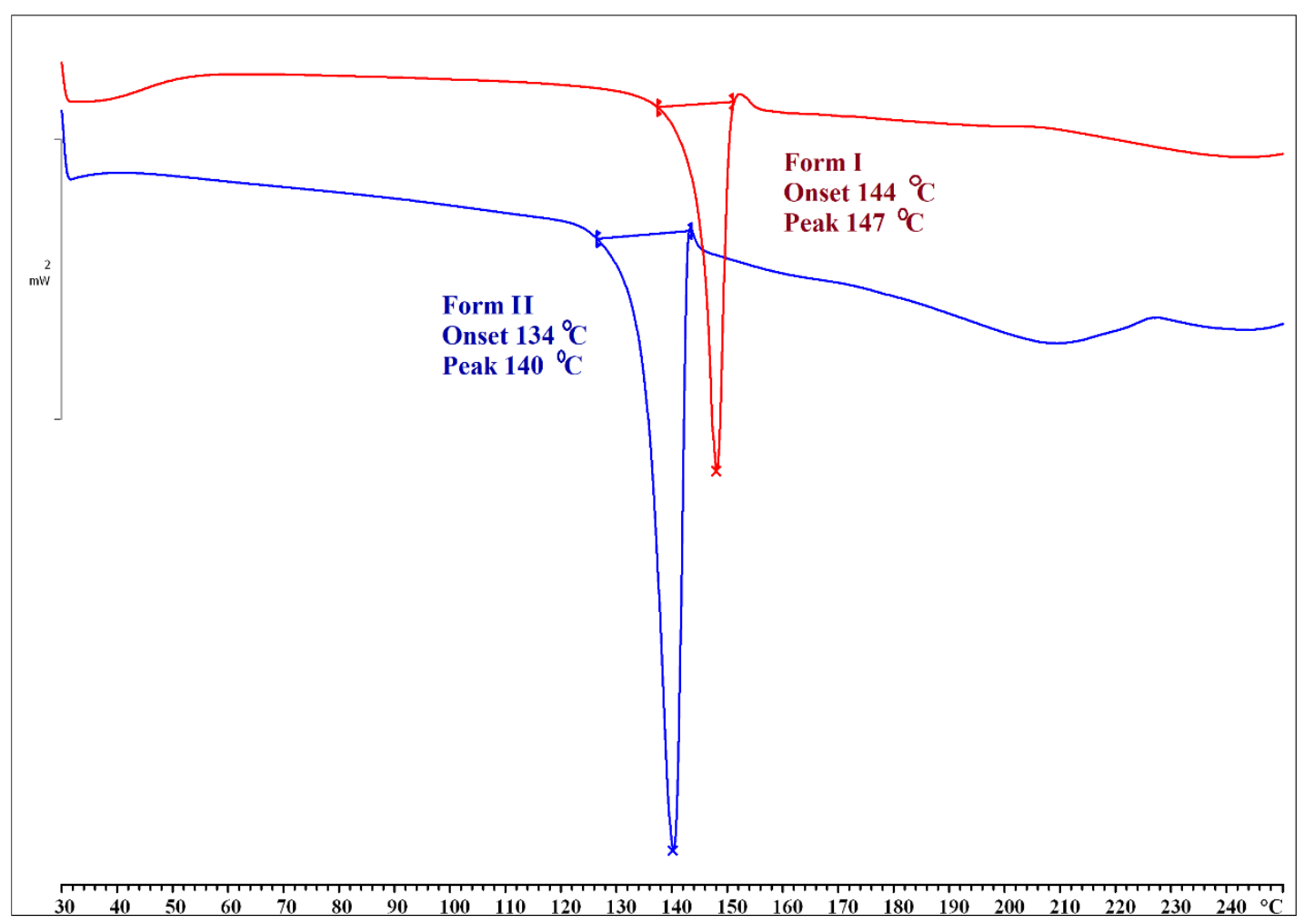

Figure S1 DSC of Form I and Form II of HCT-HP cocrystal polymorphs. Melting endotherm shows that they are monotonically related.

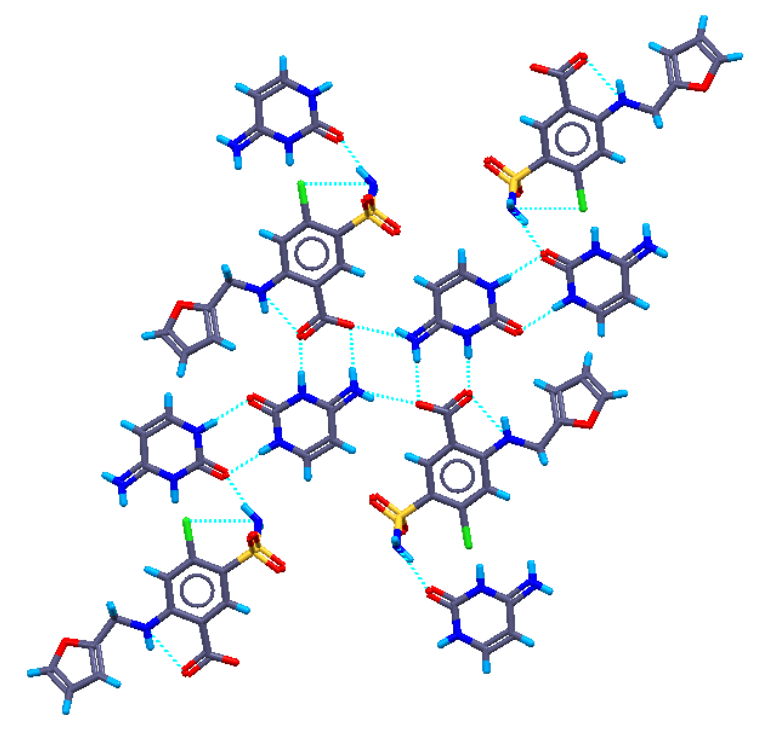

Figure S2 FUROS-CYT cocrystal structure packing, sulfonamide-syn amide and acid-pyridine synthons. 


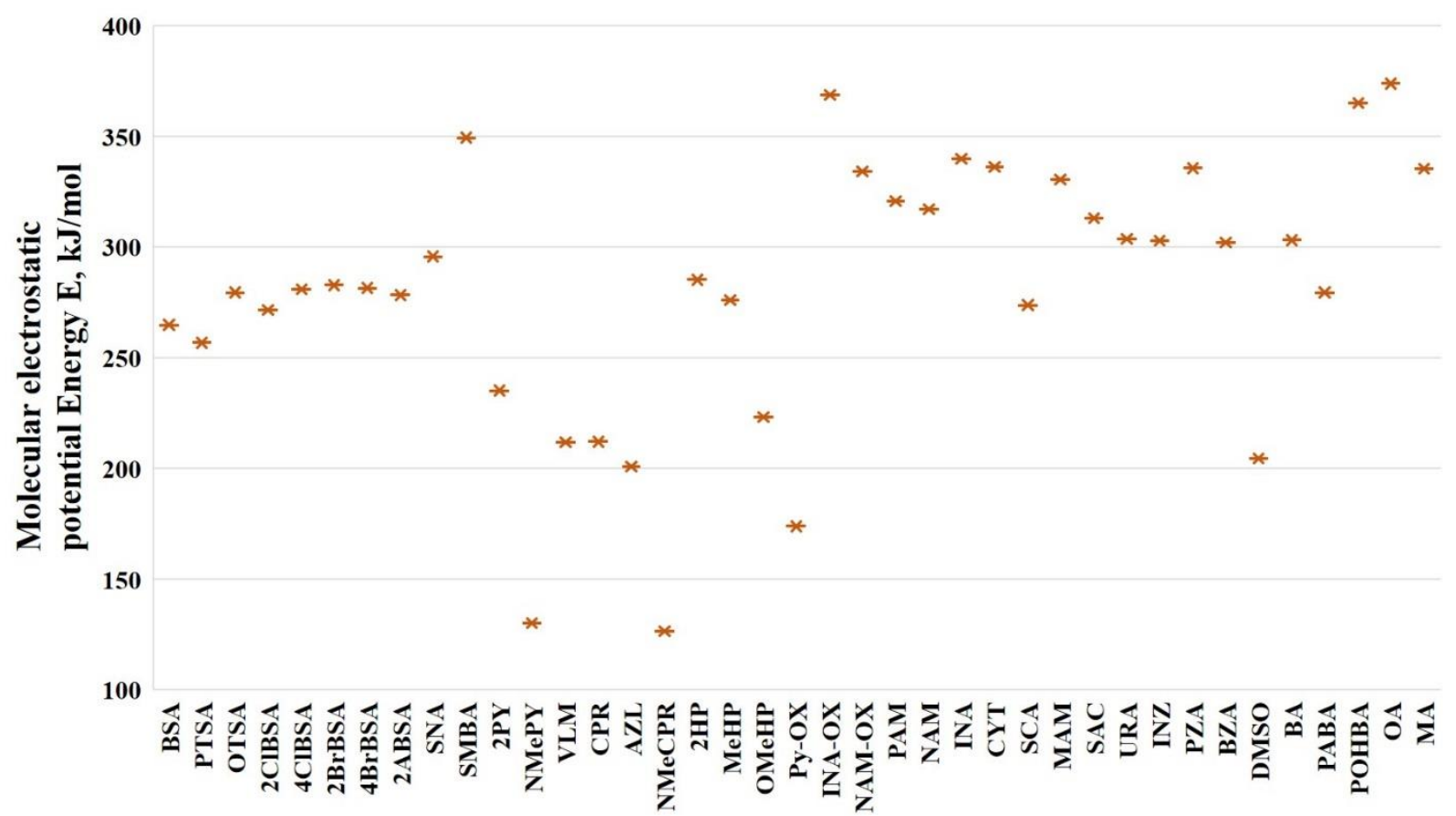

(a)

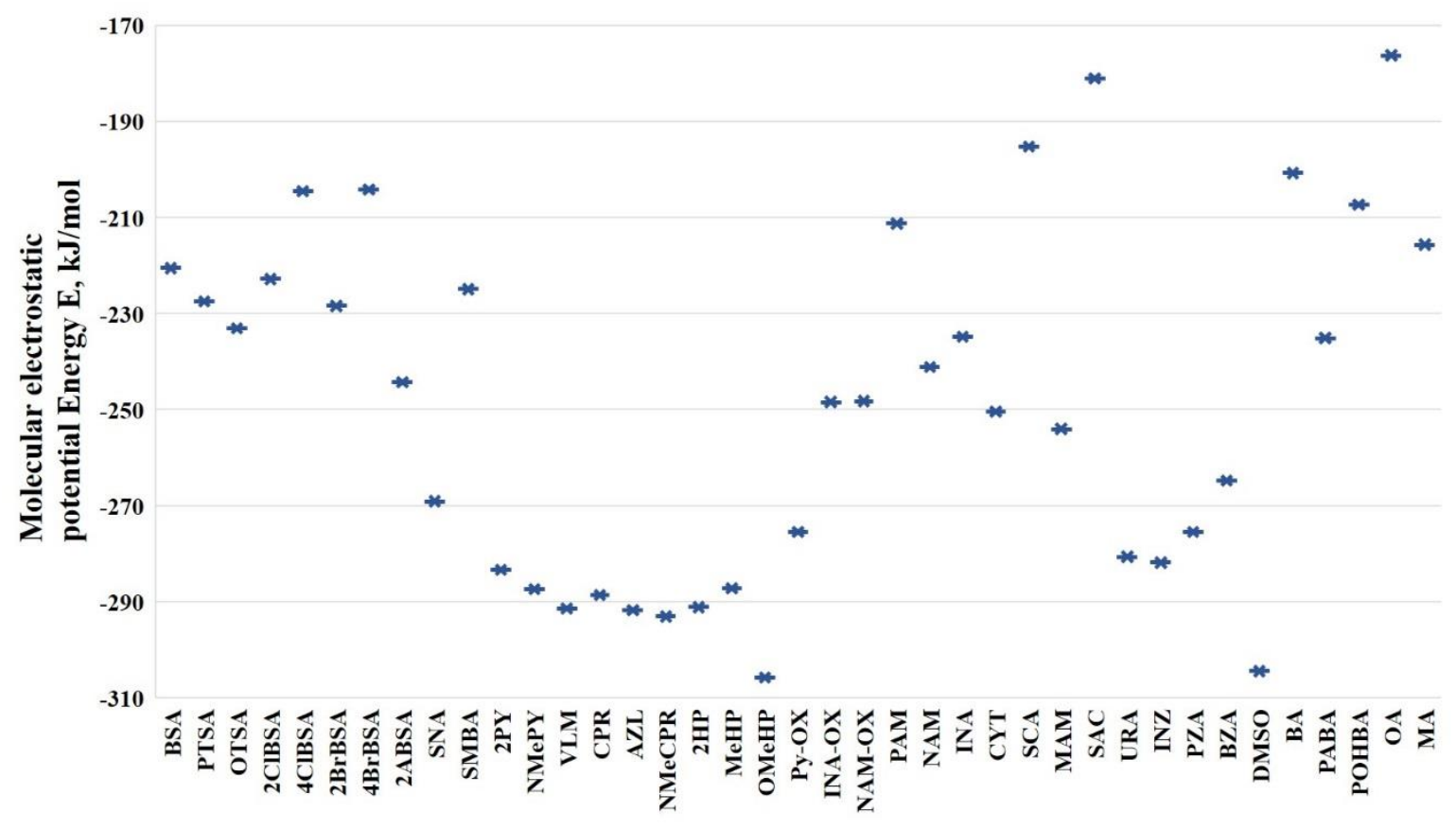

(b) 


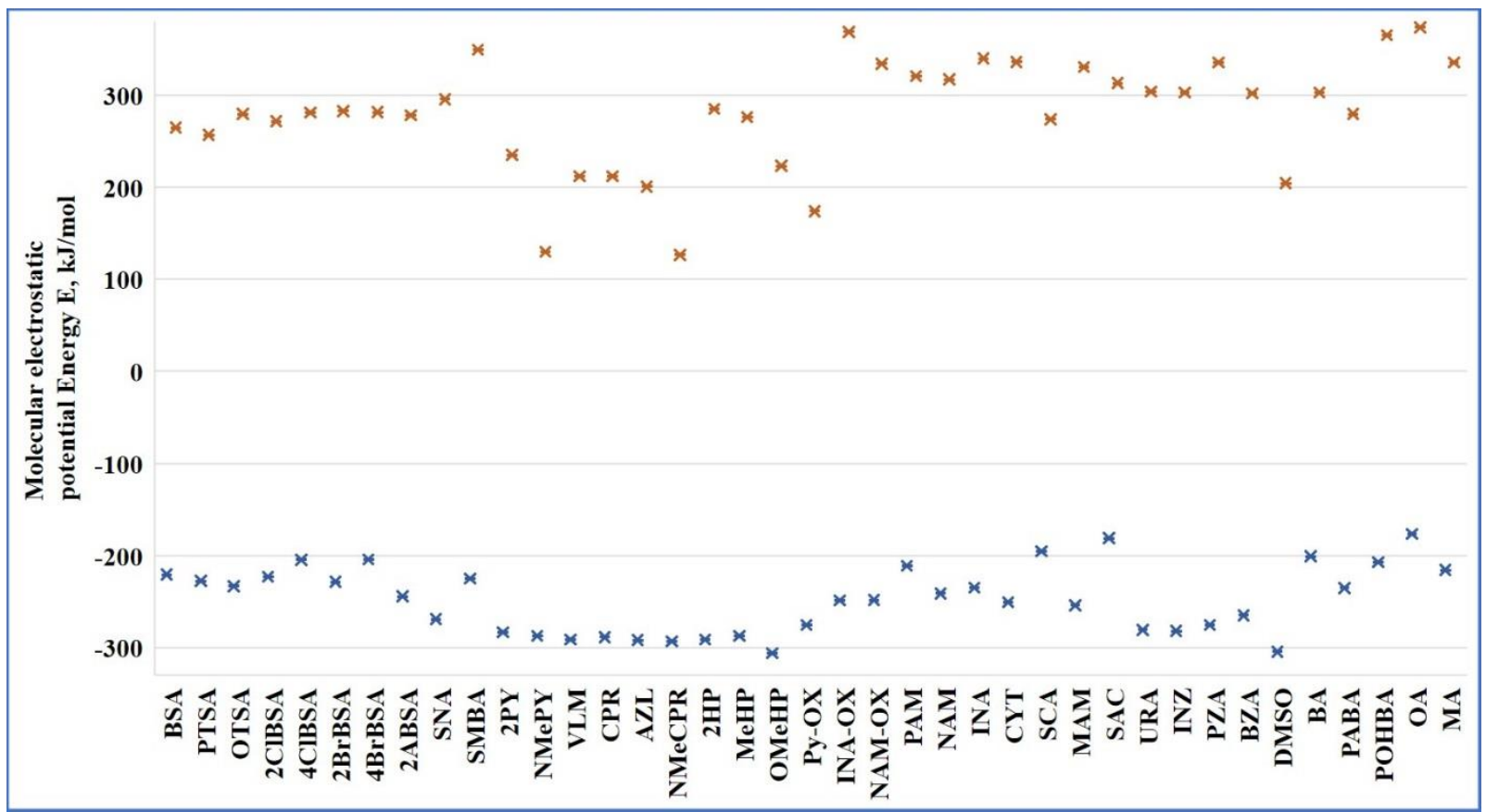

(c)

Figure S3 (a) Negative electrostatic potential (in $\mathrm{kJ} / \mathrm{mol}$ ) of all the donors and acceptors in the present study. These calculations show that lactam and syn-amide is more electronegative that $\mathrm{N}$-oxide coformers. (b) Lactam and syn-amide are less electropositive (in $\mathrm{kJ} / \mathrm{mol}$ ) compared to other coformers. (c) Comparision of the positive and negative electrostatic potential energy. 